\title{
A Comparative Study of Physical and Chemical Method for Separation of Benzoic Acid from Industrial Waste Stream
}

\author{
Rehan Khan* and Muhammad Usman \\ National University of Sciences and Technology, School of Chemical and Materials Engineering (SCME), NUST, Islamabad, Pakistan
}

\begin{abstract}
Phthalic anhydride is manufactured by the partial oxidation of ortho xylene in the presence of $\mathrm{V}_{2} \mathrm{O}_{5} / \mathrm{TiO}_{2}$ catalyst. In the residue, maleic acid, benzoic acid (BA), telluric acid and traces of other organic compounds are present in the by-product stream. There is almost $60 \%$ of $(B A)$ in the residue sample, which compels to recover the tremendous amount of (BA), as it is of great importance. The waste stream is in the form of slurry at high temperature and is solidified at room temperature. Benzoic acid was selectively removed from the solid waste of Phthalic anhydride production industry by the processes associated with less intensive parameters as compare to the extraction techniques achieved by, pressurized hot water, supercritical $\mathrm{CO}_{2}$ extraction, where the required parameters are highly intensive. The separation has been performed by using physical and chemical techniques. In physical methods recrystallization (RE), liquid-liquid extraction (LLE) as well as recrystallization followed by liquidliquid extraction (RE, LLE) and liquid-liquid extraction followed by recrystallization (LLE, RE) have been studied. In another attempt chemical derivatization (CD) has been performed for the purification purposes. The operation has been performed at the atmospheric pressure with operating temperatures of $100^{\circ} \mathrm{C}$ and $25^{\circ} \mathrm{C}$ in physical processes $(R E)$ and (LLE) respectively while in chemical derivatization it ranges from $72^{\circ} \mathrm{C}$ to $100^{\circ} \mathrm{C}$. The characterization of the samples was accomplished by GCMS, FTIR and also its melting point was determined. Almost $99 \%$ of the purity was achieved by CD, LLE, RE followed LLE and LLE followed by RE.
\end{abstract}

Keywords: Recrystallization; Liquid liquid extraction; Chemical derivatization; Reflux; Esterification; Chromatogram; Ethyl acetate; Sodium benzoate; Methyl benzoate; Benzoic acid; Phthalic anhydride

\section{Introduction}

Phthalic anhydride was first produced in 1830 by Augusta Laurent [1]. Naphthalene was the first feedstock and then o-xylene was used as feedstock after the world war for commercial production. Another feed stock can be (n-pentane). The n-pentane related process is still in development stages and requires cheap material. Yield, cost, availability are considered in the feed stocks selection. Naphthalene and $n$ - pentane are expensive as compare to o-xylene. Actually the feed for Phthalic anhydride production has been converted from naphthalene to o-xylene. In petrochemical industry, catalytic cracking is one of the fundamental process in which the heavy hydrocarbons are broken down to light hydrocarbons. The light hydrocarbons are usually of more importance. One of the examples of such process is the partial oxidation of o-xylene in the presence of air in a fixed bed reactor to produce Phthalic anhydride. It is catalytic process in which Vanadium oxide based on Tatiana is used as a catalyst. The surface area of this catalyst is $10 \mathrm{~m}^{2} / \mathrm{gram}$ and this catalyst can also be supported on ceria and silicon carbide. The major units in Phthalic anhydride plants are distillation column, Heat exchangers, vessels, heaters, compressors pumps, and cooling system. Air and o-xylene are feed to heater for the vaporization. The fixed bed reactor is suitable in the process with the operating parameters of 1-3 bar pressure and 300 to $400^{\circ} \mathrm{C}$ temperatures. Cooling is achieved by the heat exchangers for lowering the temperature. The instant removal of the heat avoids hot spots and deactivation of the catalyst. The ratio adjustment of o-xylene to air is an essential step to avoid the explosion. This process is highly exothermic in nature with $(-264 \mathrm{kcal} / \mathrm{mole})$ heat released [2-4].

The upper limit of air in the xylene feed stream should not exceed $1 \%$ for the safe operation. Large amount of benzoic acid is produced as a side reaction in the synthesis of phthalic anhydride by the partial oxidation of $o$-xylene. Reaction between oxygen and o-xylene is exothermic in nature and due to insufficient oxidation of o-xylene and rise in temperature of the reaction from $240^{\circ} \mathrm{C}$ to $480^{\circ} \mathrm{C}$ is observed with some of the side reactions [2]. Along with benzoic acid maleic anhydride, methyl maleic anhydride-toluic acid and some unknown impurities are produced in the side reactions. The series of reactions occur above the operating temperature.

\section{Main reaction}

Benzoic acid is an essential chemical with wide applications in chemical industries. It serves as food additive, medicines, cosmetics, antiseptics and also widely used in the synthesis of phenol. Environmental pollution, as a source of increasing concern might be reduced by application of proper methods of purifications aimed at reduced pressure and temperature. It is therefore essential to develop techniques that are environmental friendly, safe, effective, economical and practical. To utilize the resources and reduce the pollution, several techniques for purifying these compounds have been developed [5].

One of the ways is pressurize hot water extraction of benzoic acid (BA) and phthalic anhydride (PA) (Figure 1) from industrial waste through modified supercritical fluid extractor. It is for the first time used for the extraction of BA and PA. BA and PA can be selectively removed from the petrochemical waste by pressurize hot water extractor technique. The design is based on semi-continuous extraction. The selective extraction of BA and PA is achieved from the

*Corresponding author: Rehan Khan, National University of Sciences and Technology, School of Chemical and Materials Engineering (SCME), NUST, Islamabad, Pakistan, Tel: +923439727788; E-mail: rk054331@gmail.com

Received October 08, 2016; Accepted November 01, 2016; Published October 03, 2017

Citation: Khan R, Usman M (2016) A Comparative Study of Physical and Chemical Method for Separation of Benzoic Acid from Industrial Waste Stream. J Adv Chem Eng 7: 161. doi: 10.4172/2090-4568.1000161

Copyright: ( 2016 Khan R, et al . This is an open-access article distributed under the terms of the Creative Commons Attribution License, which permits unrestricted use, distribution, and reproduction in any medium, provided the original author and source are credited. 


\section{O-xylene is oxidized to phthalic anhydride}

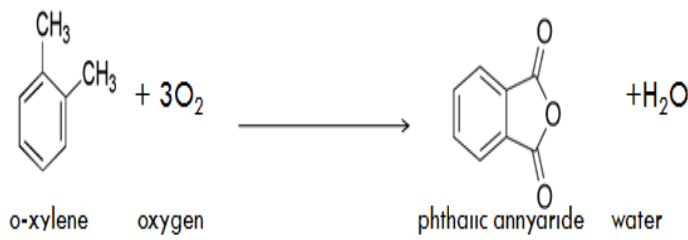

Figure 1: O-xylene is oxidized to phthalic anhydride.

mixture of phthalic acid, aldehydes, toluic acid, maleic acid and other minor impurities. The pressure, temperature, flow rate and extraction time required are $60-220$ bar, $60-140^{\circ} \mathrm{C}, 0.2-1 \mathrm{ml} / \mathrm{min}, 5-45 \mathrm{~min}$ respectively. Required parameters for PHWE purification procedure:

Temperature: $140^{\circ} \mathrm{C}$

\section{Pressure: 118 bar}

Flow rate of water: $0.9 \mathrm{ml} / \mathrm{min}$

\section{Extraction time: $29 \mathrm{~min}$}

Supercritical $\mathrm{CO}_{2}$ has also been used for the purification in the same circumstance. Variables such as temperature $\left(35-95^{\circ} \mathrm{C}\right)$, pressure (100-300 bars), static time (0-20 min), dynamic time (10-90 $\mathrm{min})$, and flow rate $(0.2-1 \mathrm{~mL} / \mathrm{min})$ were used $[6,7]$.

Recrystallization is the physical method of eliminating the impurities from the crude sample of BA. It relies on the difference in solubility of solutes in a solvent. The impurities would either be soluble in solvent at low or high temperature but the present practice is more concerned with the second phenomenon. Recrystallization is one of the physical methods for the elimination of impurities up to some extent by processing the impure sample of benzoic acid. There are two types of impurities: those more soluble in a given solvent than the main component and those less soluble. If there are any impurities that have the same solubility as the main component, then a different solvent needs to be chosen. The combination of solute and solvent should allow the dissolution in an amount that is not unduly large. The boiling point of the solvent must be lower so that it can be readily removed it from crystals. The suitability of the solvent for the recrystallization of benzoic acid is checked by determining the dielectric constant of the solvent, the property that measures the polarity of solvents. The solvent with 2-3 range value (dielectric constant) is considered non-polar while those with high than 10 are polar in nature. The solvent with 3-10 range of value are termed as intermediate polar. At $96^{\circ} \mathrm{C}$ the solubility of the benzoic acid is $67 \mathrm{gm} / \mathrm{lit}$ in water and thus making water the best solvent for this process [8]. The solvent should not have the tendency to react chemically with the purifying sample. The benzoic acid would show less solubility (Figure 2) at room temperature than at high temperature. The purification is achieved by dissolving some of the sample in a suitable solvent. Thus, if the dissolution of the sample is achieved by heating, the crystals will appear after cooling the heated solution. The extent of precipitation relay on the difference in solubility of the substances in solvent at two extreme ranges of temperature. The upper limit is the boiling point of the solvent while the lower is determined by the experimental convenience. Acetones ethyl alcohols, and other organic compounds can also be used for the same purpose but water is more cost-effective. Compounds, which are less soluble, will crystallize first.
The crystallization process itself helps in the purification because as the crystals form, they select the correct molecules, which fit into the crystal lattice and ignore the wrong molecules. This is of course not a perfect process, but it does increase the purity of the final product [9]. Liquidliquid extraction (LLE) is one of the separating technique in which one or more solute in feed is transfer to another immiscible liquid. It is one of the frequent separating techniques employed to isolate one or two components from the mixture. It is very versatile method of great value not only in laboratory but in the daily life. In technical sense the principle of the extraction is based on the equilibrium distribution of substance in the two immiscible phases, one of which is an extracting solvent $[9,10]$. The solvent needs to be pure but with the tendency to react selectively with the component of interest. The mass transfer phenomenon is dominated in the operation. It is the way of extracting compounds from solids or liquids by liquid solvent. The main principle of the process is the difference in solubility of solute in two different immiscible liquids. In the present case ethyl acetate is an appropriate solvent for the dissolution of sample [11]. The solubility of the benzoate ion is marked more in water at low temperature as compare to ethyl acetate, thus the mass transfer of benzoate ions into the aqueous phase is dominated. Dielectric constant value of the ethyl acetate is 6.02 that put it in the range of intermediate polar solvent with the suitability for the dissolution of benzoic acid. The general method is to use the extracting solvent in small amount than using it in bulk at once. Better extraction was achieved when the experiment was performed by the addition of solvent in several steps. It is used in the condition when distillation process in uneconomical or insufficient to extract the pure of components. It is appropriate method in azeotropic mixture separation and heat sensitive components. Along with some advantages, the slow and tedious nature of the LLE and the utilization of large amount of solvent for the extraction are the problems associated with LLE [12] (Figure 3).

Chemical derivatization $(\mathrm{CD})$ is one of the significant techniques for decontamination of impure sample of benzoic acid. The process is

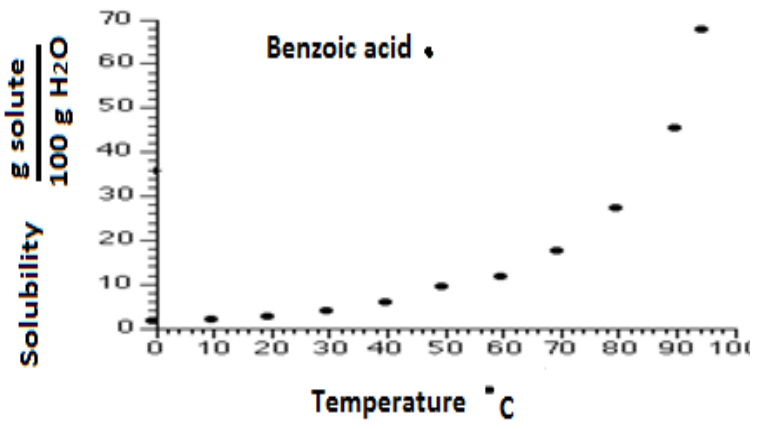

Figure 2: Benzoic Acid Solubility Curve in Water (Solvent).

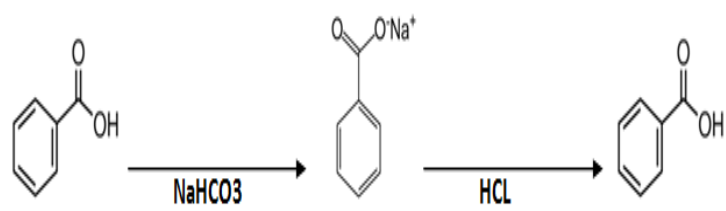

Benzoic acid step 1 sodium benzoate step 2 benzoic acid 
also termed as esterification. The selected route is about the conversion of benzoic acid to the methyl benzoate by the reaction of methanol with benzoic acid under reflux, the product from this reaction is reacted with base $\mathrm{NaOH}$ under reflux to form sodium benzoate. The protonation of the sodium benzoate is the last step of the purification process [1316]. These reactions are commonly catalysed by concentrated and small amount of an acid that speeds up the reaction between the two reactants. The Keq equilibrium constant for these reactions is 4 for the most of the reactions and thus the ratio of the two reactants is $1: 1$ with almost $67 \%$ yield [17]. Excess of alcohol can enhance the yield by shifting the equilibrium to the right side of the equation. The process is also called fisher esterification reaction. The yield may reach to the $95 \%$ by the use of excess amount of alcohols. The recovery achieved was too low as compared to the other (Figures 4 and 5) purifications process [18-20].

\section{Materials and Methods}

Water was used as solvent in the recrystallization, Ethyl acetate acted as a solvent in the ionization of benzoic acid in LLE while the $\mathrm{NaHCO}_{3}$ was used in the production of sodium benzoate and HCL used for protonation purpose. In the chemical derivatization the methanol is used in the production of methyl benzoate from benzoic acid in the presence of $\mathrm{H}_{2} \mathrm{SO}_{4}$ as catalyst. The transformation of methyl benzoate from the aqueous to phase is achieved by ether, $\mathrm{MgSO}_{4}$ as a drying agent while $\mathrm{NaOH}$ used for the production of sodium benzoate to make benzoic acid after the protonation of sodium benzoate.

\section{Recrystallization}

The recrystallization was performed by taking 5 gram of crude benzoic acid sample and put in the $500 \mathrm{~mL}$ of water. Heat the flask until the crude sample is dissolve and the solution becomes clear. Sometimes the sample have some impurities that are not soluble in water and are visible, the addition of more solvent is avoided. The usage of solvent should be minimum. After heating, cooling step is significance to acquire pure crystals. The cooling rate alters the purity of the crystals. The slower the cooling, the slower is the formation of crystals but with excellent purity. An inverse relation exists between rate of cooling and purity Excellency. Sometimes the crystals yield is less and can be increased by scratching the sides of beaker with glass rod. The crystals obtained are filtered and washed with distilled water several times [8].

Recovery=weight of purified sample / weight of benzoic acid in the crude sample $\times 100$

Initial weight taken $=5$ gram

Yield $=4.25 / 5=85 \pm 5 \%$

Recovery of benzoic acid assuming $60 \%$ in crude sample is almost $95 \pm 3$ but the presences of impurities were detected.

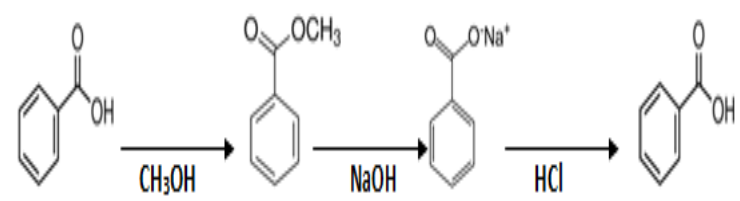

Benzoic acid Methylbenzoate Sodium benzoate Benzoic acid

Figure 4: Describe the Summary of the Process.

\section{Liquid-liquid extraction}

The liquid-liquid extraction was initiated by the dissolution of 5 gram of crude benzoic acid in the ethyl acetate as an organic solvent in separating funnel. Benzoic acid is soluble in the medium polar solvent with less toxicity. Extraction is accomplished by shaking the solution with second solvent in the separating funnel. $\mathrm{NaHCO}_{3} 50 \mathrm{ml}$ of 1 molar solution was added to the separating funnel in order to convert the benzoic acid to sodium benzoate and transfer it to the aqueous phase from organic phase. The reason of transformation of sodium salt from organic to aqueous phase is the difference in the solubility. The ionic compound would have markedly different solubility from the acids. The mixture is shacked up to 2 minutes for each batch of the added aqueous solvent [10]. The carbon dioxide produced is vent off the separating funnel. The shaking is done until no $\mathrm{CO}_{2}$ is produced. The aqueous phase with sodium benzoate has more density than the organic phase layer, thus after each step the lower layer is placed in the beaker for the protonation process. Sometimes the entire product is not extracted in single operation and the process should be repeated twice or more for the better recovery. The protonation of the sodium benzoate is done by the addition of $20-25 \mathrm{~mL} \mathrm{HCl}$ ( 3 molar). After the protonation, the white precipitate are obtained that are washed with distilled water during filtration phase. The whole process is about the conversion of benzoic acid to sodium benzoate and again this product to purified benzoic acid with overall $90 \%$ recovery and $99 \%$ purity $[11,12]$.

Recovery=weight of purified sample/ weight of benzoic in the crude sample $\times 100$

Initial weight taken $=5$ gram

Yield $=2.7 / 5=54 \pm 4 \%$

Recovery of benzoic acid by assuming $60 \%$ in the crude sample $=90 \%$

\section{Recrystallization followed by liquid-liquid extraction}

The recrystallization followed by liquid-liquid extraction was performed for the purification. The combinations of these two kinds of processes one after another, yield benzoic acid with appreciating purity. The recovery of the benzoic acid was little bit less as compare to the (LLE) method when performed alone. The total recovery is $48 \%$

Recovery=weight of purified sample/ weight of the crude sample $\times 100$

Initial weight taken $=5$ gram

Yield $=2.4 / 5=48 \pm 3 \%$

Recovery of benzoic acid by assuming $60 \%$ in the crude sample $=80 \%$

\section{Liquid-liquid extraction follower by recrystallization}

The techniques, liquid-liquid extraction followed by recrystallization were performed for elimination of the unwanted impurities associated with benzoic acid. The same procedure was followed for both the process. Almost the same recovery and purity was achieved as in (RE, LLE) process.

Recovery=weight of purified sample/ weight of benzoic acid in crude sample $\times 100$

Initial weight taken $=5$ gram

Yield $=2.45 / 5=49 \pm 3 \%$

Recovery of benzoic acid by assuming $60 \%$ in the crude sample $=81 \%$ 


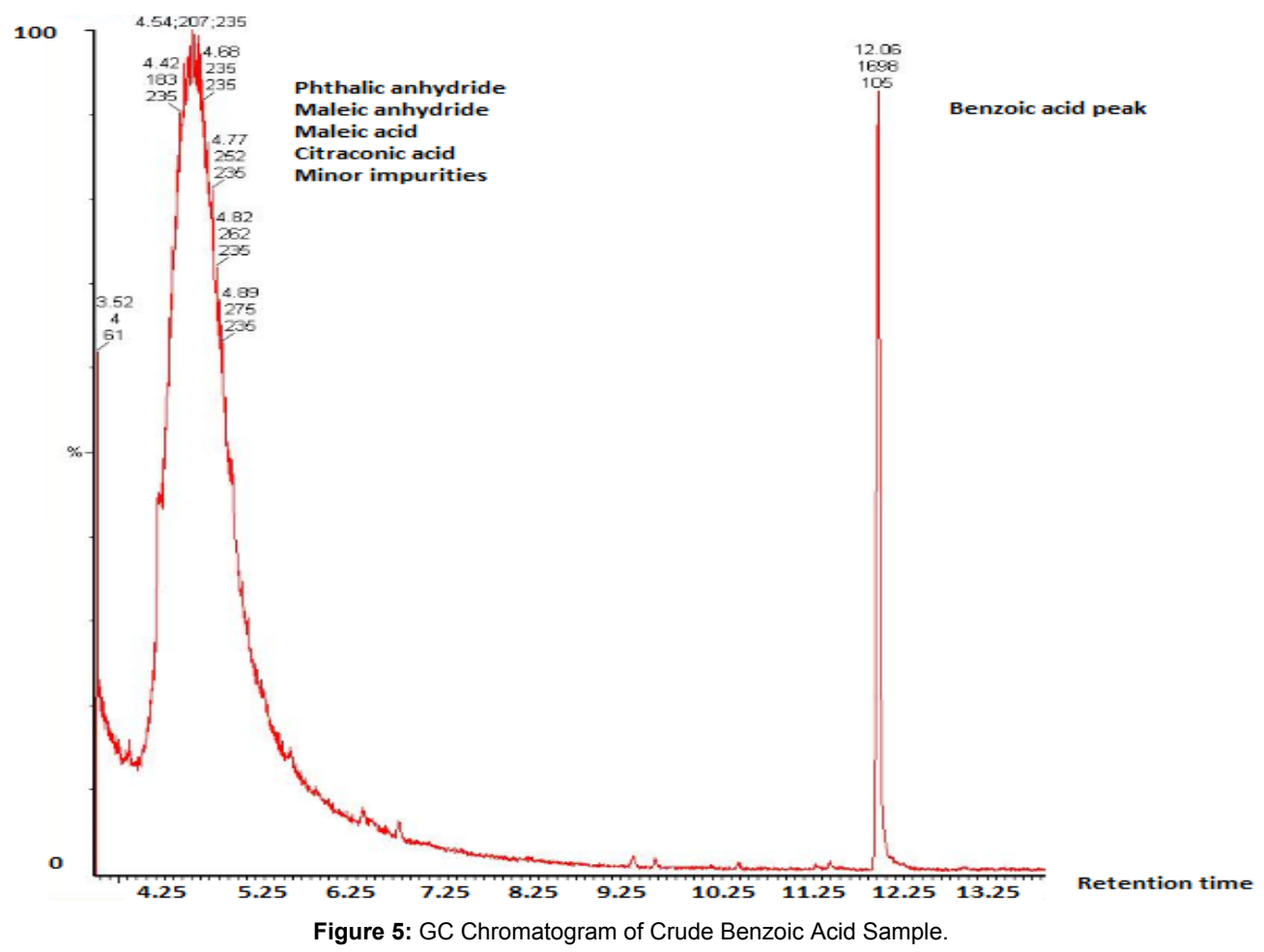

\section{Chemical derivatization}

In this attempt the purification is achieved by performing the chemical method. This process is about the conversion of benzoic acid to the methyl benzoate, the product is then converted to sodium benzoate and the protonation of the sodium benzoate is the last step of the purification process. The initiation of chemical derivatization is made by taking 5 grams of crude benzoic acid and mixed with $45 \mathrm{ml}$ of methanol in the round bottom flask. The amount of methanol can be varied but the suitable amount in this case is $25 \mathrm{ml}$. The reaction is catalysed by the addition of $1.2 \mathrm{ml} \mathrm{H}_{2} \mathrm{SO}_{4}$. The reactants are mixed and attached to the reflux condenser. Reaction under reflux condenser is the way to maintain constant elevated temperature $[17,18]$. One is the reaction flask usually round bottom flask and the other part is a condenser attached to the reaction flask. During heating the solvent evaporates and then come down back to the reacting flask after being cooled by the circulating water. In this process the volume of the solvent does not change because no solvent is allowed to go out of the flask. The cooling is done by the circulating water around the condenser. Most of the organic reactions do not take place at room environment so the reflux heating adjustment becomes necessary for it i.e., the reaction between benzoic acid and methanol. The mixture is heated almost for 3 hour by keeping temperature at $64^{\circ} \mathrm{C}$. After reflux the reactants, methyl benzoate and some of the unreacted benzoic acid and sulfuric acid were poured in the separating funnel. It is mixed with $75 \mathrm{ml}$ water for the washing purpose. Rinsing the reaction flask with $35 \mathrm{ml}$ ether is done and then poured into the separating funnel too. After shaking the lower aqueous layer is drained that contained unreacted methanol and acid. The remaining ether layer is washed with $15 \mathrm{ml}$ water and drained. The ether layer is again washed with $\mathrm{NaHCO}_{3}(0.5 \mathrm{M})$ solution to remove the untreated benzoic acid. During this washing cautiously shaking is done due to the release of $\mathrm{CO}_{2}$ gas in the process can create pressure inside the separating funnel. Methyl benzoate is retained and the ether is evaporated by rotary evaporator at $36^{\circ} \mathrm{C}$. The methyl benzoate obtained was dried by the drying agent. The removal of water in these types of reactions is necessary because it may alter the rate of reaction. During the reaction between methyl benzoate and sodium hydroxide, the presence of water associated with methyl benzoate may change the required concentration of the sodium hydroxide solution. The methyl benzoate is then refluxed with $\mathrm{NaOH}$ saturated solution at $100^{\circ} \mathrm{C}$ with almost the same volume as methyl benzoate. The reactants are refluxed for 3 hour and the stopped [19]. Sodium benzoate is produced in this process which is then converted to benzoic acid by the addition of 3 molar HCL to the sodium benzoate almost double the volume of it. The precipitation occurs that is the conformations of the presence of benzoic acid.

Recovery=weight of purified sample / weight of benzoic acid in crude sample $\times 100$

Initial weight taken $=5$ gram

Yield $=1.75 / 5=35 \pm 4 \%$

Recovery of benzoic acid by assuming $60 \%$ in the crude sample $=58 \%$

\section{Results and Discussion}

\section{Characterization of the samples}

The characterization of the purified and crude sample has been performed by melting point apparatus, GC-MS and FTIR analytical equipment's.

\section{Melting point}

The temperature of solid at which its state changes from solid state to liquid state is called melting point. Difference substances have different melting points (Table 1). Determining the melting point is one of the methods of inspecting about the purity of the substance. 
For example the melting point of $\mathrm{BA}$ is $122.4^{\circ} \mathrm{C}$ in the literature but in the impure state the value of melting is lesser than the literature value. It is actually the physical property of compound. The difference in the melting points of compounds is due to the different structure. Melting point is insensitive to pressure and no pressure correction is needed while in the boiling point it matters. Melting and boiling point can be useful in the identification of substance [20].

\section{GC-MS result}

It is an analytical instrument combining gas chromatography with ability of separation, with mass spectrometry ideal in elucidation structure of separated components. This technique is usually used for the volatile organic components. In these kinds of equipment's there exists equilibrium between stationary phases, which is responsible for the separation. The carrier gas is usually an inert gas which carries the solute through column. This carrier gas is also called mobile phase $\left(\mathrm{N}_{2}, \mathrm{He}\right)$ with $1-2 \mathrm{ml} / \mathrm{min}$ flow rate. Physical and chemical natures of molecules make them to travel with different speed in a column [20-25].

In the chromatogram (Figure 6) the broad peak of the impurities appears in the range of 4.75 to 6.75 min retention time while the benzoic acid peak is at the $11.08 \mathrm{~min}$ of retention time with low intensity. The sample along with the impurities was tested by GC-MS. The chromatogram indicates the presence of impurities like maleic acid, toluic acid, Pimide and other unknown organic compounds etc. The small peak of the benzoic acid is the evidence of the less amount of benzoic acid in the crude sample.

The sample purified by the recrystallization is not that much pure as compare to the sample purified by the other purifying methods. Recrystallization is not the better choice to purify the sample but in combination with liquid-liquid extraction and chemical derivatization is the best choice to get high purity. The sample purified by the recrystallization method shows the result of BA with high peak intensity in the chromatogram as compare to the peak representing impurities. Benzoic acid in the GC chromatogram can be identified by its retention time. The retention time varies for different components. Retention time of benzoic acid is almost 11 minutes. Similarly the study of MS can also leads to know about the compound under observation.

The Figures 7-11 are the chromatograms of the purified samples indicating almost $99 \%$ purity. In the GC chromatogram of these purified samples there is no such peak of impurities but only one peak of benzoic acid with more intensity.

\section{FTIR Results}

FTIR is a powerful technique for the identification of pure organic and inorganic compounds. The interactions of radiations occur with those substances which possess permanent or induced dipole moments and excitation of vibrational state exist. The discrete level of vibrational energy should be in these kinds of molecules. It actually detects the vibration characters of different chemical functional groups. The compound must have dipole moment during vibration. Dipole moment is the property that is related to net measure of molecules polarity $[26,27]$. The FTIR spectrum of the crude sample is different from the purified samples while the sample purified by (RE) and the crude sample can be distinguished from the literature spectrum. In the spectrum the $2500-3500 \mathrm{~cm}^{-1}$ is the region indicates the $(\mathrm{OH})$ stretching bond, 3000 indicates the presence of $\mathrm{C}-\mathrm{H}$ stretching bond related to the aromatic ring, $1715 \mathrm{~cm}^{-1}$ range shows the presence of $\mathrm{CO}$ while the $1622-1496 \mathrm{~cm}^{-1}$ is the range of the aromatic ring $[28,29]$.

The spectrum of the crude sample and the sample purified by (RE) have different spectrum as compare to the literature spectrum, because of the presence of a lot of $\mathrm{OH} 2500-3000 \mathrm{~cm}^{-1}, \mathrm{CH}_{3} 2875 \mathrm{~cm}^{-1}, \mathrm{CH} 3000$ $\mathrm{cm}^{-1}$ and other types of groups associated with the impurities (Figures 12-14). The spectrums that are represented by the following Figures 1517 resemble the literature spectrum of the benzoic acid. The reason of the resemblance is the absence of a lot of $(\mathrm{OH}) 2500-3000 \mathrm{~cm}^{-1},\left(\mathrm{CH}_{3}\right)$ $2875 \mathrm{~cm}^{-1},(\mathrm{CH}) 3000 \mathrm{~cm}^{-1}$ and other types of groups associated with the impurities $[30,31]$.

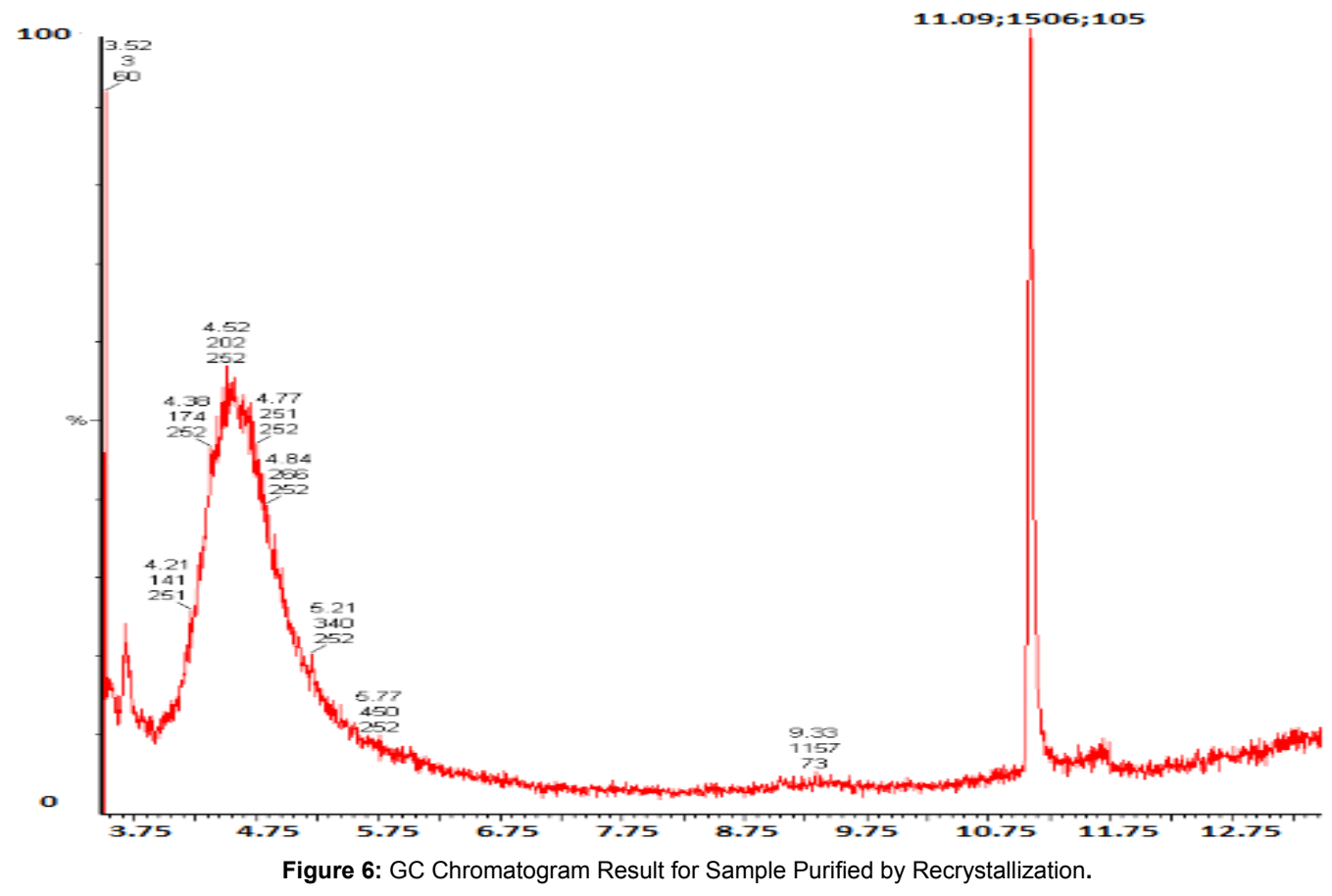




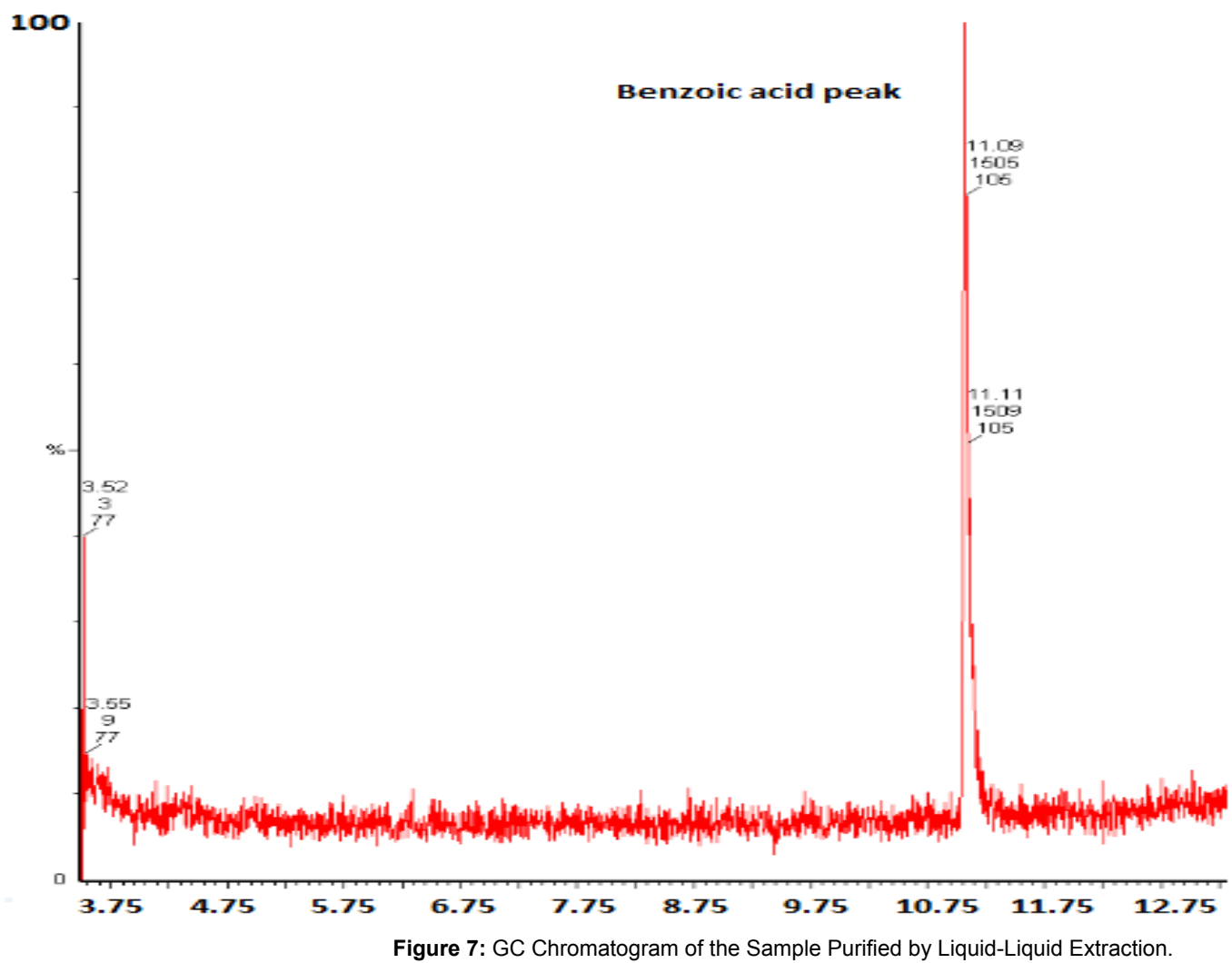

Retention time

100

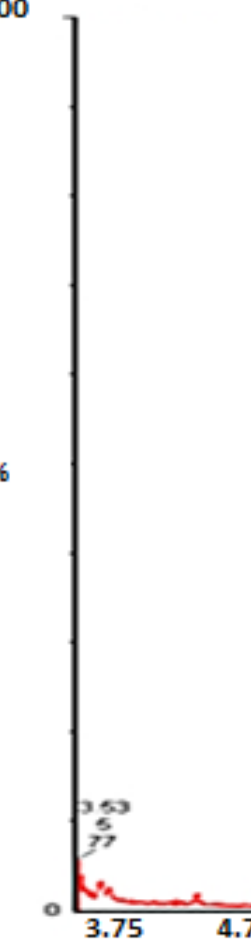

$11.10 ; 1508 ; 105$

Retention time 
Citation: Khan R, Usman M (2017) A Comparative Study of Physical and Chemical Method for Separation of Benzoic Acid from Industrial Waste Stream. J Adv Chem Eng 7: 161. doi: 10.4172/2090-4568.1000161
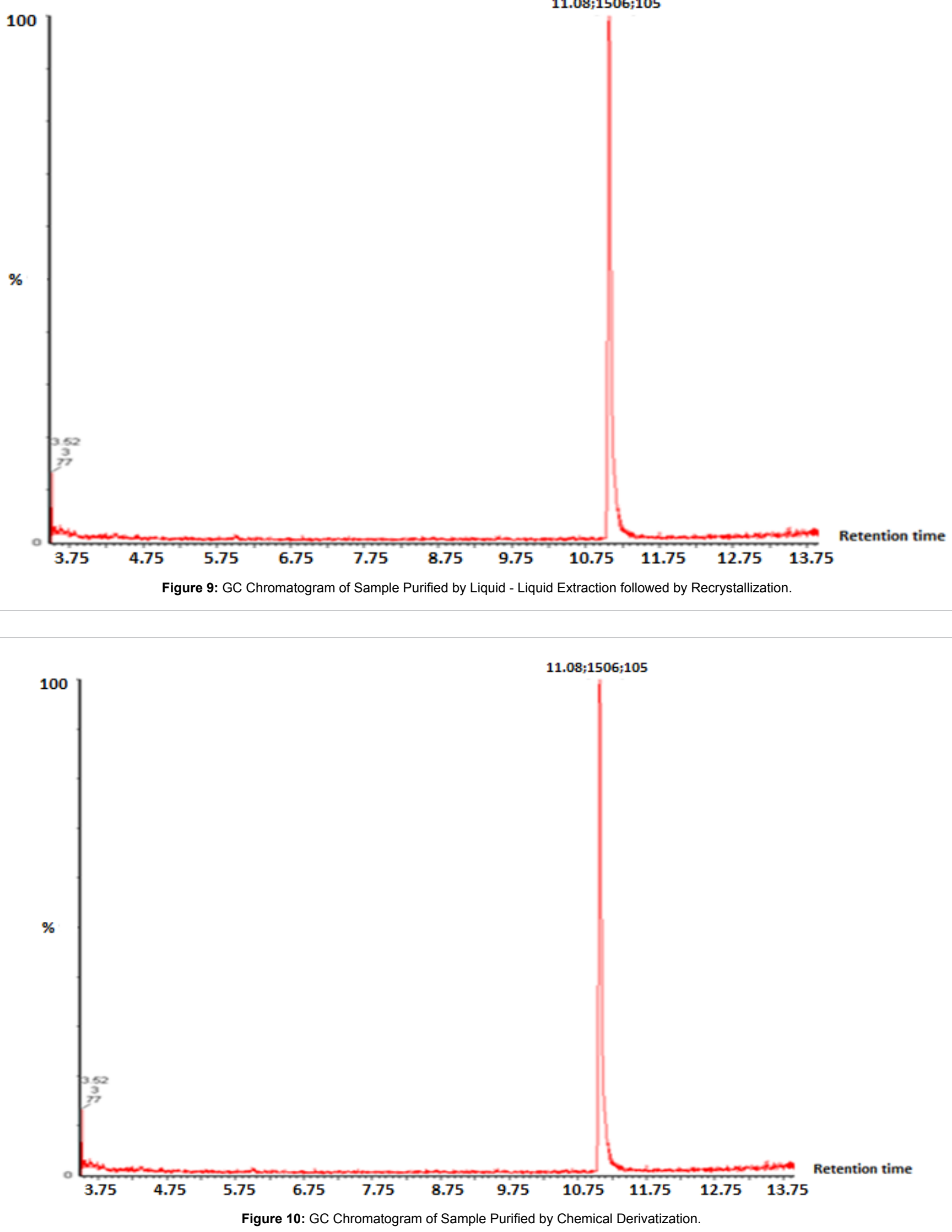


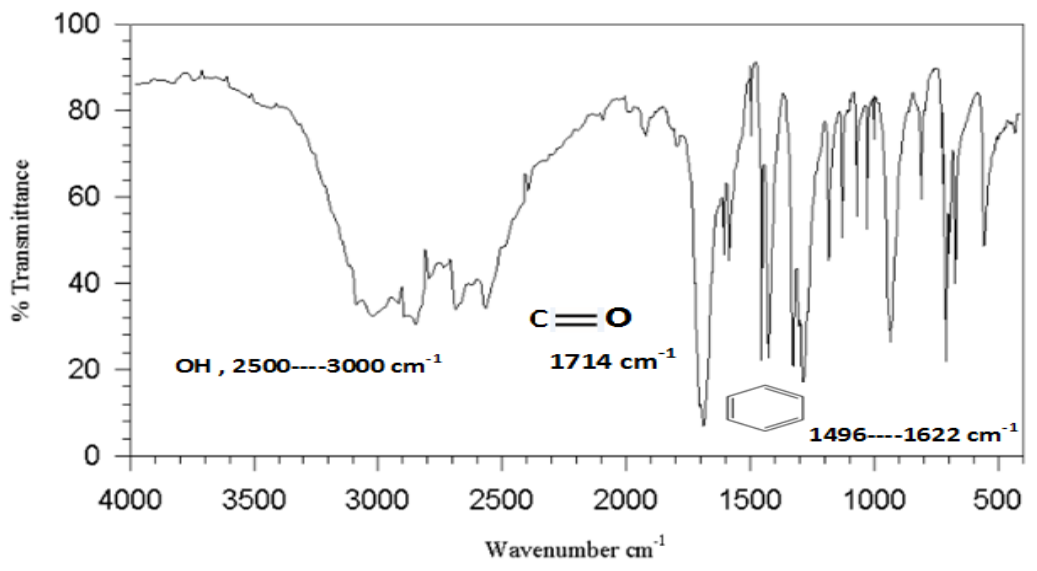

Figure 11: Literature FTIR Spectrum of the Benzoic Acid.

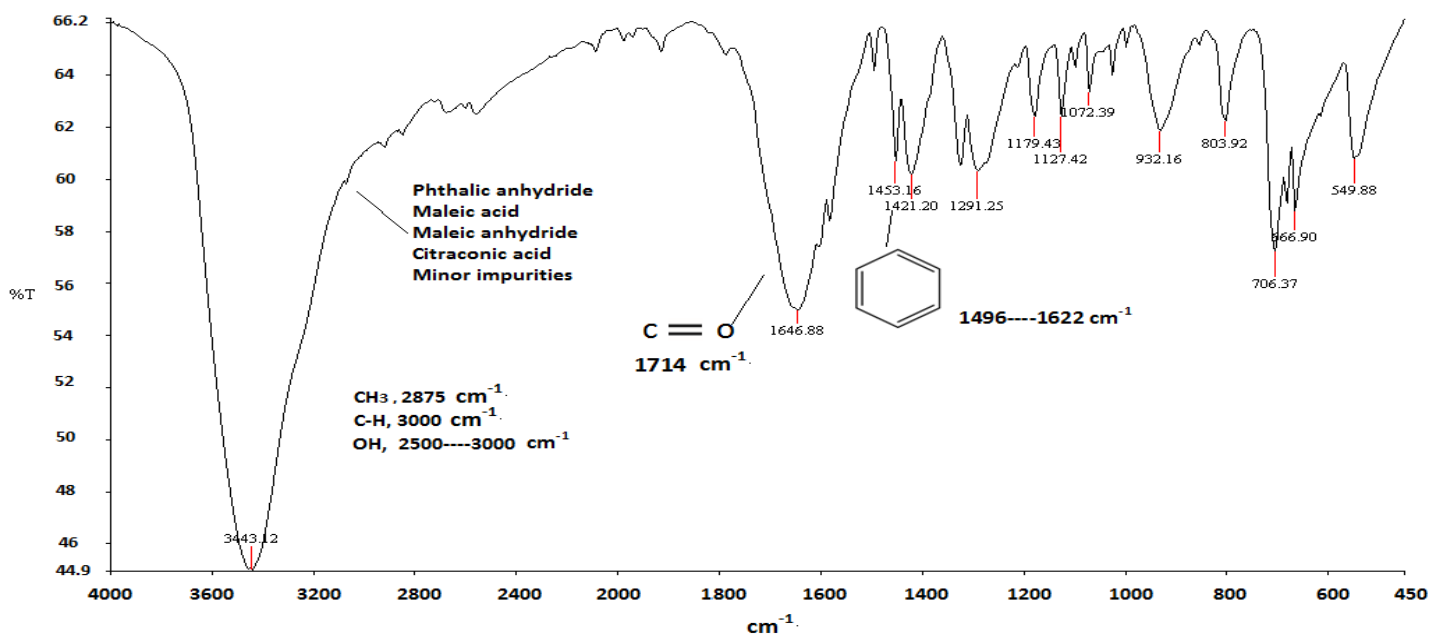

Figure 12: FTIR Spectrum of Crude Benzoic Acid.

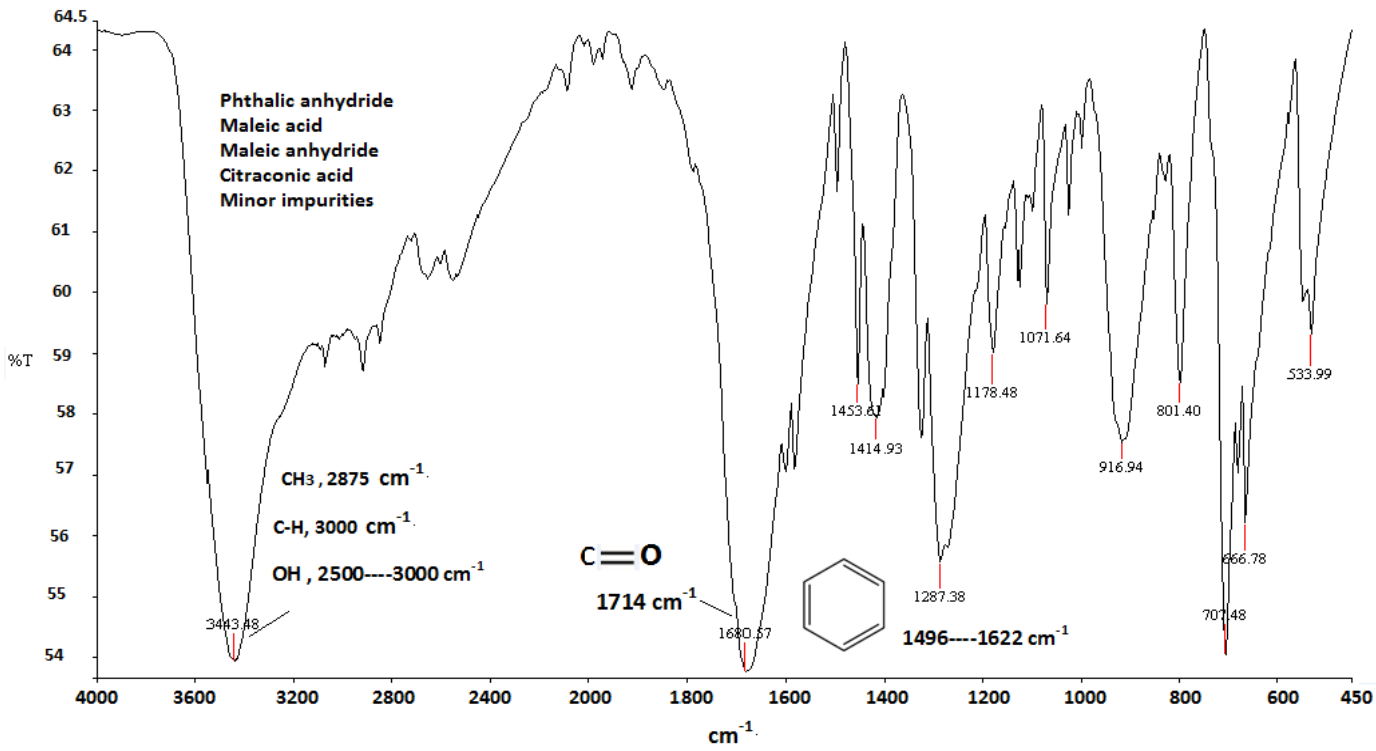

Figure 13: FTIR Spectrum of the Sample Purified by Recrystallization. 


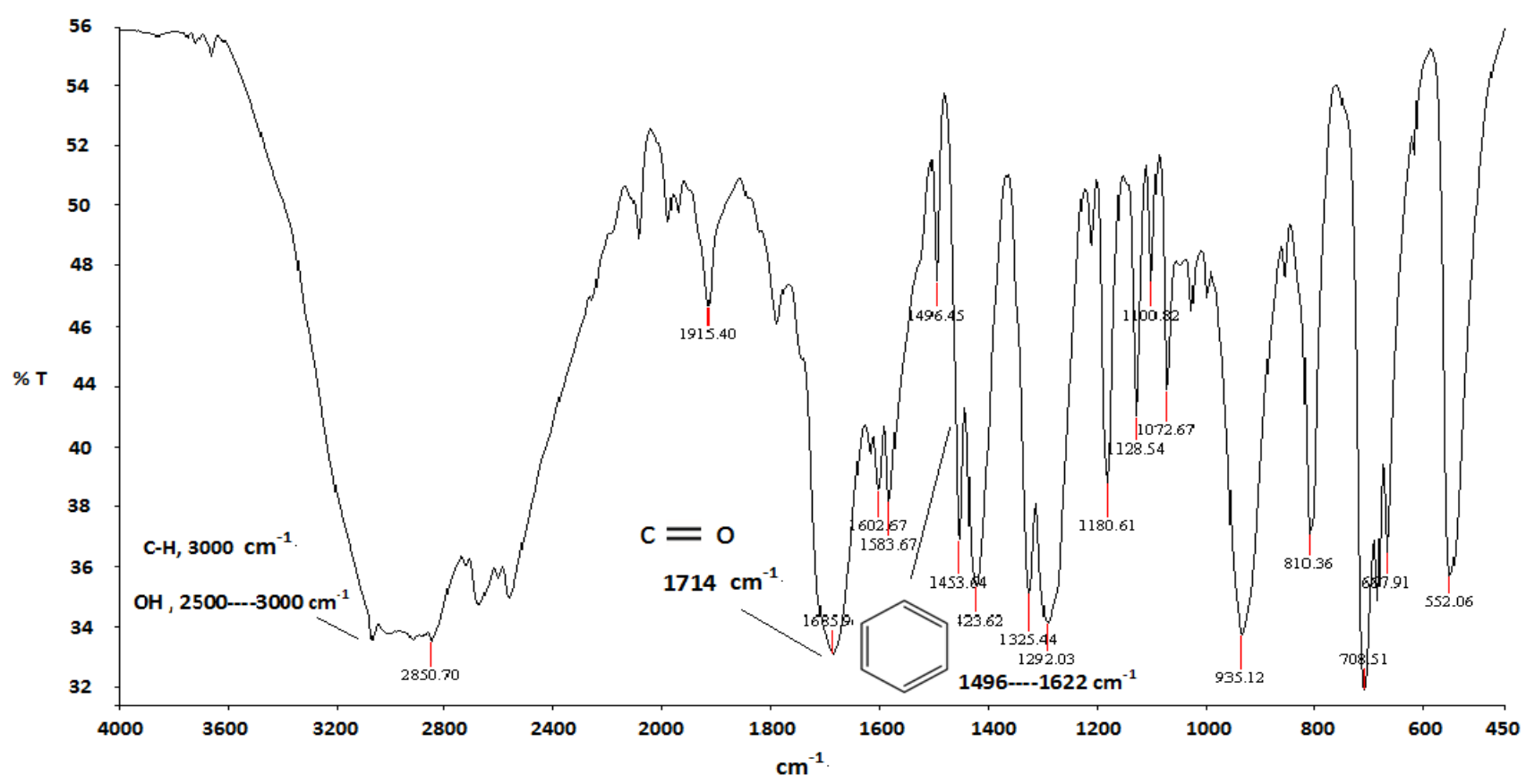

Figure 14: FTIR Result of the Sample Purified by Liquid-Liquid Extraction.

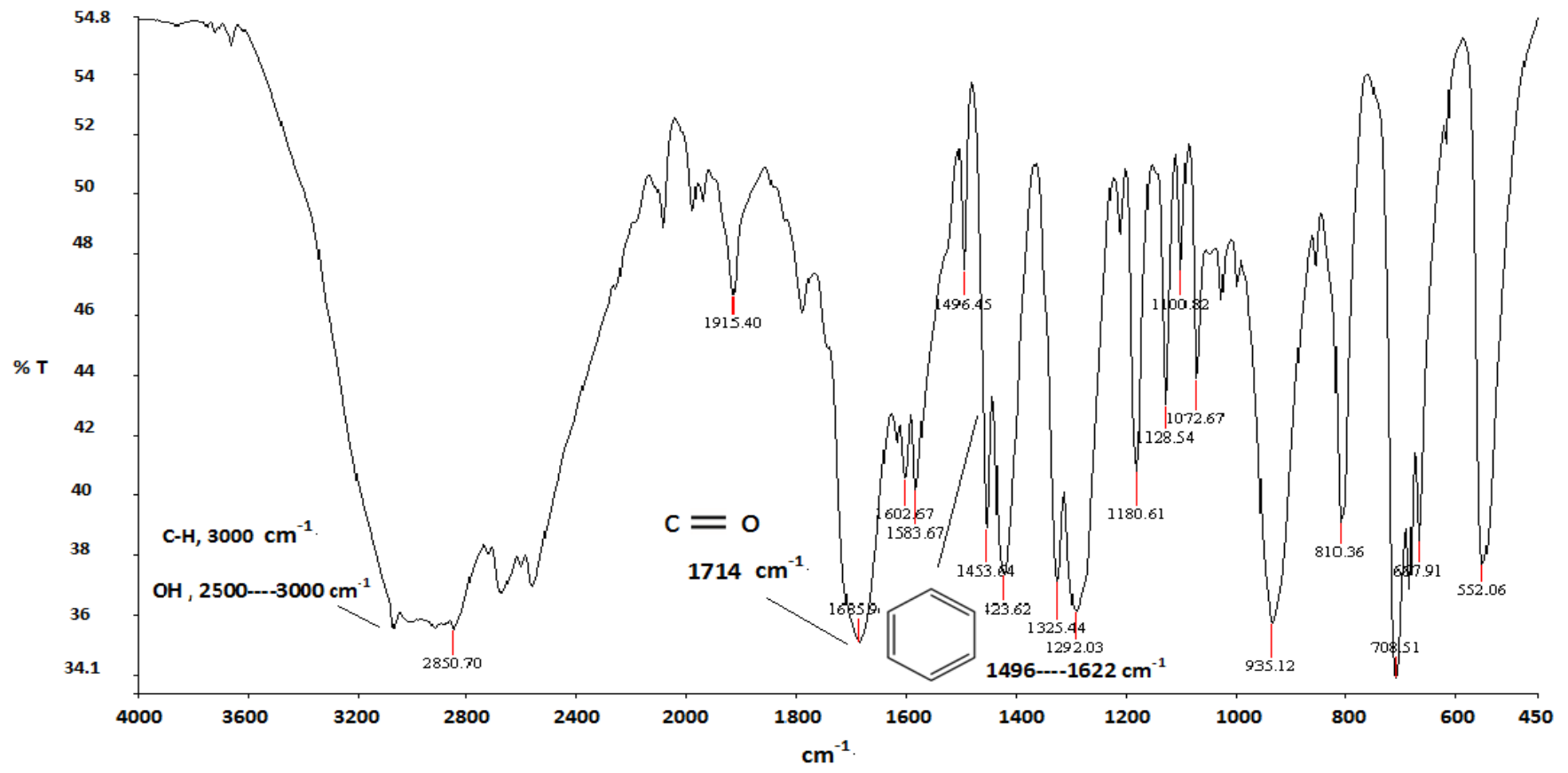

Figure 15: FTIR Result of the Sample Purified by Recrystallization followed by Liquid - Liquid Extraction.

\section{Conclusion}

Experiments illustrate a clear view about the recovery and purity of the impure benzoic acid. The purification accomplished by (RE) yield BA with $85 \pm 5$ yield with recovery but the cause of not considering it a best way is the incomplete elimination of impurities from the sample of the as shown in the GC chromatogram. The peak associated with the impurities can be clearly observed at 3.75-5.75 min range of retention time.
(LLE) is considered the best way of decontaminating the sample with $54 \pm 4 \%$ yield and (BA) recovery of $90 \pm 2 \%$ with outstanding purity. The Figure 6 indicates a clear intensive peak of the only BA almost at the retention time of $8 \mathrm{~min}$ after it is injected.

(RE) followed by (LLE) had almost $48 \pm 3 \%$ yield with $80 \pm 3$ recovery of (BA). And (LLE) followed by (RE) had $49 \pm 3 \%$ yield with 81 $\pm 2 \%$ recovery of (BA) with appreciating effects on the purity of crude 


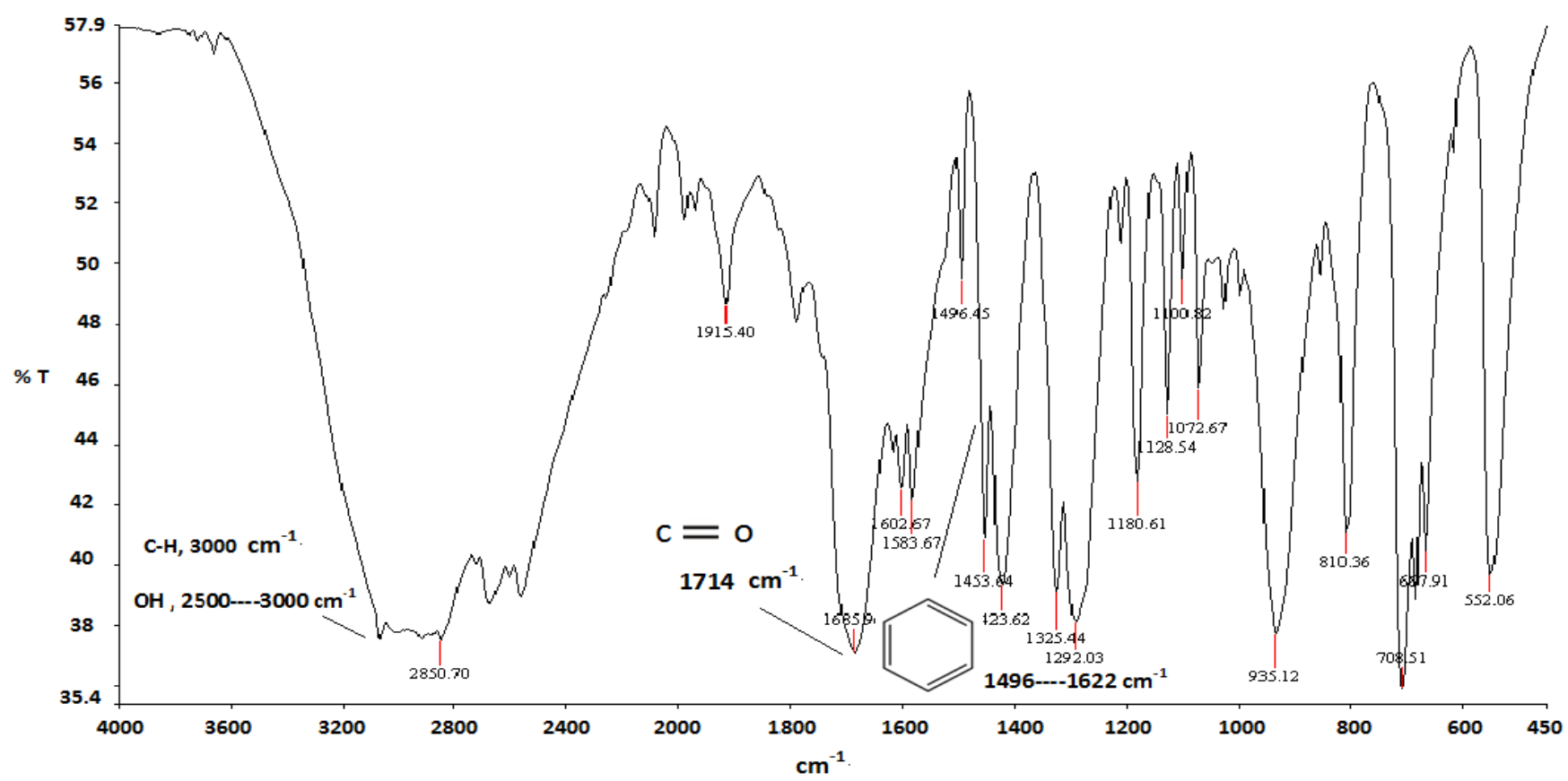

Figure 16: FTIR Result of the Sample Purified by Liquid - Liquid Extraction followed by Recrystallization.

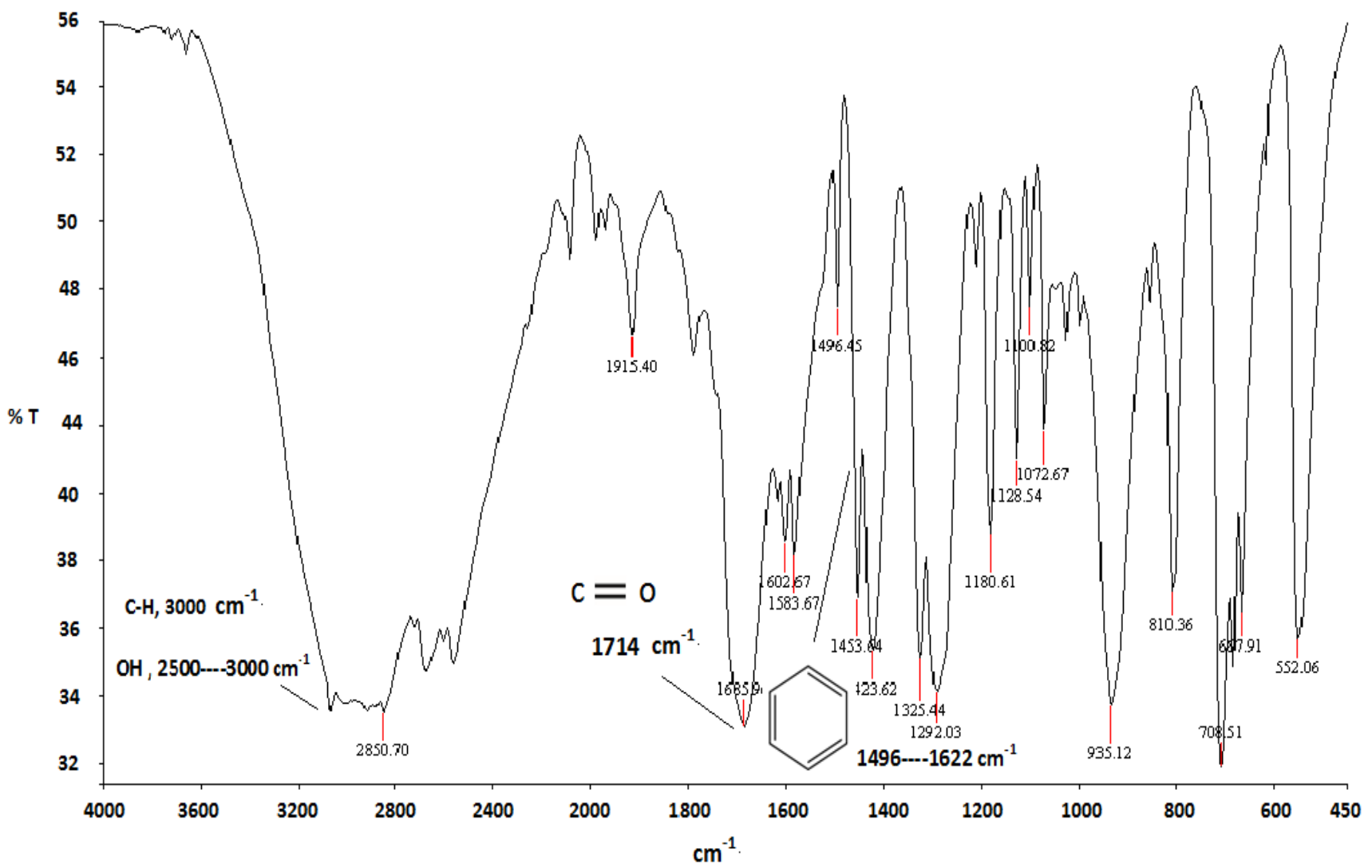

Figure 17: FTIR Result of the Sample Purified by Chemical Derivatization. 
Citation: Khan R, Usman M (2017) A Comparative Study of Physical and Chemical Method for Separation of Benzoic Acid from Industrial Waste Stream. J Adv Chem Eng 7: 161. doi: 10.4172/2090-4568.1000161

Page 11 of 11

\begin{tabular}{|c|c|}
\hline Purification processes & Melting point \\
\hline Literature melting point of benzoic acid & $122.4^{\circ} \mathrm{C}$ \\
\hline Crude benzoic acid & $112.1 \pm 1^{\circ} \mathrm{C}$ \\
\hline Sample purified by Re-crystallization & $121.1 \pm 1^{\circ} \mathrm{C}$ \\
\hline Sample purified by Liquid-Liquid extraction & $122.3 \pm 1^{\circ} \mathrm{C}$ \\
\hline $\begin{array}{c}\text { Sample purified by Liquid-Liquid extraction followed by Re- } \\
\text { crystallization }\end{array}$ & $122.4 \pm 1^{\circ} \mathrm{C}$ \\
\hline $\begin{array}{c}\text { Sample purified by Re-crystallization followed by Liquid-Liquid } \\
\text { extraction }\end{array}$ & $122.3 \pm 1^{\circ} \mathrm{C}$ \\
\hline Sample purified by Chemical Derivatization method & $122.1 \pm 1^{\circ} \mathrm{C}$ \\
\hline
\end{tabular}

Table 1: Melting point of the crude and purified benzoic acid.

sample. Only one peak exists, associated with the benzoic acid while the same result at the retention time of $11 \mathrm{~min}$. In the chemical method of purification, the chemical derivatization (CD) was performed, had $35 \pm 5 \%$ yield with $58 \pm 5$ recovery of (BA) but leads us to high purity.

The significance of these purifying techniques is its association with less intensive operating parameters i.e., low temperature and pressure as compare to the extraction achieved by vacuum distillation, pressurized hot water, supercritical $\mathrm{CO}_{2}$ extraction techniques, where the required parameters are too intensive.

\section{References}

1. Blondel M (2001) Auguste Laurent and alkaloids. History of medicines and pharmacy (France) 49: 303-314.

2. Anastasov $\mathrm{Al}$ (2003) Deactivation of an Industrial $\mathrm{V}_{2} \mathrm{O}_{5} / \mathrm{TIO}_{2}$ Catalyst for the Oxidation of O-Xylene into Phthalic Anhydride. Chemical Engineering and Processing, Process Intensification 42: 449-460.

3. Frank $P$ (1938) Phthalic anhydride production. Google patent.

4. Gimeno (2008) Selective Oxidation of O-Xylene to Phthalic Anhydride over $\mathrm{V}_{2} \mathrm{O}_{5} / \mathrm{TIO}_{2}$ Kinetic Study in Fluidized Bed Reactor. Chemical Engineering and Processing, Process Intensification 47: 184.

5. Dias CR, Portela MF, Galan-Fereres M, Banares MA, Granados ML, et al. (1997) Selective oxidation of O-xylene to phthalic anhydride on $\mathrm{V}_{2} \mathrm{O}_{5} / \mathrm{TIO}_{2}$ coated $\mathrm{SiO}_{2}$. Catalysis letters 43: 117-121.

6. Teo CC, Tan SN, Yong JW, Hew H, Ong ES (2010) Pressurized hot water extraction. J Chromatogrm 1217: 2484-2494.

7. Kamali H, Ghaziaskar HS, Khakshour A, Kaboudvand M (2013) Supercritical $\mathrm{CO}_{2}$ extraction of phthalic anhydride, benzoic acid and maleic acid from petrochemical wastes. Journal of Supercritical 74: 46-51.

8. Thomas CL (1970) Catalytic Process and Proven Catalysts. Academic Press, New York, USA, pp: 182-184.

9. Farrauto RJ, Bartholomew CH (1997) Fundamentals of Industrial Catalytic Processes, Chapman \& Hall, London. 621-639.

10. Baumann JB (1979) Solvent Selection for Recrystallization: An undergraduate organic experiment. Journal of Chemical Education 56: 64.

11. Andrea A, Patrick L, Heider, Nopphon W, Klavs F, et al. (2013) MembraneBased, Liquid-Liquid Separator with Integrated Pressure Control. American Chemical Society (ACS) 52: 10802-10808.

12. Rezaee M, Assadi $Y$, Milani H, Mohammad R, Aghaee E, et al. (2006) Determination of Organic Compounds in Water Using Dispersive Liquid-Liquid Micro extraction. Journal of Chromatography 1116: 1-9.

13. Donald L, Pavia P (2004) Introduction to Organic Laboratory Techniques. Thomson Brooks/Cole, pp: 312-314.

14. Kantlehner W (1991) Comprehensive Organic Synthesis: Selectivity, Strategy and Efficiency in Modern Organic Chemistry. In: Trost BM, Fleming I (eds.) Pergamum Press Inc., Elmsford, NY, USA 6: 485-599.

15. Johnson RW, Fritz E (1989) Fatty Acids in Industry. Marcel Dekker Inc., New York, USA.

16. Cardoso L, Neves SCG, Silva M (2008) Esterification of oleic acid for biodiesel production catalysed by $\mathrm{SnCl}_{2}$ : A kinetic investigation. Energies 1: 79-92.

17. Cardoso L, Natalino R, Silva M (2010) Bioenrgy II: tin catalyzed esterification of free fatty acids. International Journal of Chemical Reactor Engineering 8: 1-12.

18. Nelson PE, Ekanayake (1994) A process for the manufacture of ester by esterification of carboxylic acid with alcohols accompanied by bubbling. Chem Abstr 121: 925.

19. Krebecheck LO (1993) Esterification of carboxylic acid with alcohols involving the use of sulfuric acid as catalyst and desiccants. Chem Abstr 121: 925.

20. Berghund $\mathrm{P}$, Honquist M, Hult K, Hoegberg H (1995) Alcohols as enantio selective inhibitors in a lipase catalyzed esterification of a chiral acyl donor. Biotechnol Lett 17: 55-60.

21. Bhatnagar R, Arivid R, Bhatia S (1994) Esterification of N-Butanol with Butyric Acid Using Amberlyst-15 (Macro Porous) Resin as Catalyst. A kinetic study. Indian Chem Eng 36: 120-123.

22. Lewis RJ (2004) Sax's Dangerous Properties of Industrial Materials. Wiley Interscience 11: 1-29.

23. Grob G (1977) Modern Practice of Gas Chromatography, In: Robert L (ed.) John Wiley \& Sons.

24. Amirav A, Gordin PA, Alon M, Fialkov AB, Poliak M, et al. (2008) Gas Chromatography Mass Spectrometry with Supersonic Molecular Beams. Journal of Mass Spectrometry 43: 141-163.

25. Sloan K, Mustacich M, Eckenrode RV (2001) Development and evaluation of a low thermal mass gas chromatograph for rapid forensic GC-MS analyses. Field Analytical Chemistry \& Technology 5: 288-301.

26. McLafferty FW, Hertel RH, Villwock RD (1974) Probability based matching of mass spectra, rapid identification of specific compounds in mixtures. Organic Mass Spectrometry 9: 690-702.

27. Paula PA, Julio de (2009) Elements of physical chemistry. 5th edn. Oxford, UK, p: 459.

28. Ricco B (2015) An opto-electronic system for in-situ determination of peroxide value and total phenol content in olive oil. Journal of Food engineering 146: 1-7.

29. Mukamel S (2000) Multidimensional Fentosecond Correlation Spectroscopies of Electronic and Vibrational Excitations. Annual Review of Physical Chemistry 51: 691-729.

30. Kohli K, Davies GV, West D, Estreicher E, Gregorkiewicz S, et al. (2006) Isotope Dependence of the Lifetime of the $1136 \mathrm{~cm}^{-1}$ Vibration of Oxygen in Silicon. Physical Review Letters 96: 225-503.

31. Pollock HM, Chalmers DA, Griffiths PR (2002) The use of near-field probes for vibrational spectroscopy and photo thermal imaging. In: Handbook of vibrational spectroscopy. John Wiley \& Sons Ltd. 2: 1472-1492. 